\title{
Broadband SiGe/Si quantum dot infrared photodetectors
}

\author{
C.-H. Lin, C.-Y. Yu, C.-Y. Peng, W. S. Ho, and C. W. Liu ${ }^{a)}$ \\ Department of Electrical Engineering, National Taiwan University, Taipei, Taiwan 106, \\ Republic of China and Graduate Institute of Electronics Engineering, National Taiwan University, \\ Taipei, Taiwan 106, Republic of China
}

(Received 26 August 2006; accepted 30 November 2006; published online 12 February 2007)

The broadband absorption of metal-oxide-semiconductor $\mathrm{SiGe} / \mathrm{Si}$ quantum dot infrared photodetectors is demonstrated using boron $\delta$ doping in the Si spacer. The peak at 3.7-6 $\mu \mathrm{m}$ results from the intersubband transition in the SiGe quantum dot layers. The other peak at 6-16 $\mu \mathrm{m}$ mainly comes from the intraband transition in the boron $\delta$-doping wells in the Si spacers. Since the atmospheric transmission windows are located at 3-5.3 and 7.5-14 $\mu \mathrm{m}$, broadband detection is feasible using this device. The $\delta$ doping in $\mathrm{SiGe}$ quantum dots and $\mathrm{Si}_{0.9} \mathrm{Ge}_{0.1}$ quantum wells is also investigated to identify the origin of the absorption. (c) 2007 American Institute of Physics. [DOI: $10.1063 / 1.2433768]$

\section{INTRODUCTION}

Broadband infrared detection is attractive in thermal imaging, target identification, medical, and other applications. ${ }^{1}$ Broadband photodetectors demonstrated so far are based on III-V materials. ${ }^{2,3}$ With the advantages of integration with $\mathrm{Si}$ electronics and low cost, ${ }^{4}$ the Si-based broadband detectors are highly desirable.

A quantum dot infrared photodetector (QDIP) provides the advantages of no polarization selection rule (normal incident), small dark current, and high operation temperature. Due to the Stranski-Krastanov growth mode of quantum dots (QDs), the size of dots and width of wetting layers are hardly changed. As a result, the absorption region is limited to a certain wavelength. Quantum wells (QWs) with different thickness or compositions can be fabricated to achieve multicolor detection. ${ }^{5}$ However, strain due to the misfit between $\mathrm{Ge}$ and $\mathrm{Si}$ has to be considered to avoid the formation of dislocations.

We present a metal-oxide-semiconductor (MOS) $\mathrm{SiGe} / \mathrm{Si}$ QDIP with $\delta$ doping in the Si spacer, with a broadband spectrum covering most of the 3-5.3 and 7.5-14 $\mu \mathrm{m}$ atmospheric transmission windows. The $\delta$ doping in Si spacers provides the QDs with a sufficient hole concentration and forms a $\delta$-doping well in Si. Due to the strong electric field formed by locally ionized dopants and band gap narrowing by delocalization of acceptor states in the reciprocal space, a QW is formed in the valence band of Si spacers. ${ }^{6}$ The holes in QDs could be excited by mid-wavelength infrared (3.7-6 $\mu \mathrm{m}$ detection). The $\delta$-doping QWs contribute to long-wavelength infrared detection through intraband transition (cutoff wavelength at $16 \mu \mathrm{m}$ ). Boron $\delta$ doping in QDIPs achieves broadband detection without increasing the process complexity, as compared with the complex process to incorporate SiGe QWs.

The MOS structure uses an ultrathin tunneling oxide to allow carrier tunneling under infrared excitation and to re-

\footnotetext{
${ }^{a)}$ Author to whom correspondence should be addressed; electronic mail: chee@cc.ee.ntu.edu.tw
}

duce dark current. This simple structure of the MOS detector is attractive for integration with $\mathrm{Si}$ electronics. Note that the detector should be operated in the inversion bias region to reduce dark current.

\section{DEVICE FABRICATION}

The 20-period SiGe QDs with a 2-3 nm wetting layer were grown on $100 \mathrm{~mm} p$-type (100) Si substrates with the resistivity of $15-25 \Omega \mathrm{cm}$ by ultrahigh vacuum chemical vapor deposition (UHVCVD). Due to different in situ annealing times and interdiffusion between $\mathrm{Si}$ and $\mathrm{Ge}$, the top SiGe dot with shorter annealing time after formation has a higher Ge concentration $(\sim 60 \%)$ as compared to the bottom SiGe dot $(\sim 40 \%),{ }^{7,8}$ which suffers longer annealing time after formation. The different $\mathrm{Ge}$ concentrations are due to the difference in thermal budget after growth. The Ge concentration variation reflects the broad peaks in photoluminescence and absorption measurements. A $2 \mathrm{~nm}$ layer of lowtemperature $\left(50{ }^{\circ} \mathrm{C}\right)$ oxide ${ }^{9}$ was deposited on top of the sample by liquid phase deposition (LPD) to fabricate MOS tunneling diodes ${ }^{10}$ (Fig. 1). SiGe QD layers were separated by $80 \mathrm{~nm} \mathrm{Si}$ spacer layers. Boron $\left(10^{19} \mathrm{~cm}^{-3}\right)$ was $\delta$ introduced in the middle of the growth of each $\mathrm{Si}$ spacer layer. Al was deposited on top of the oxide to form the gate electrode (with an area of $3 \times 10^{-2} \mathrm{~cm}^{2}$ ), and also deposited on the back of the sample to form the Ohmic contact.

The large valence band offset between $\mathrm{Si}$ and $\mathrm{SiGe}$ forms discrete quantum states in the SiGe QDs. Under infrared exposure, the confined holes can be excited and contribute to the photocurrent. The spectral response is measured by a Fourier transform infrared (FTIR) spectrometer (PerkinElmer Spectrum 2000) coupled with a cryostat and an SR570 current preamplifier. The incident light is unpolarized and the devices are under normal incidence.

To further study the absorption mechanism, two other samples with the same $\delta$-doping profile are also measured for comparison (Table I). The " $\delta$-spacer" and " $\delta$-QD" samples have the same QD structure, but with different $\delta$-doping locations. The former is in the Si spacer, while the latter is in 


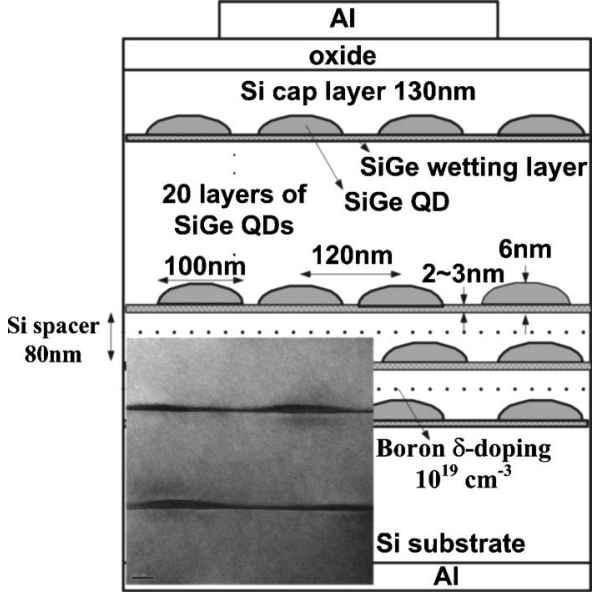

FIG. 1. The structure of the MOS SiGe/Si QDIP. The 20-layer SiGe quantum dots were formed by ultrahigh vacuum chemical vapor deposition, and the $\delta$ doping was introduced in the Si spacer. The inset shows the TEM photograph of SiGe quantum dots.

the SiGe QD layer. The " $\delta$-SiGe01" sample has five-period 7-nm-thick $\mathrm{Si}_{0.9} \mathrm{Ge}_{0.1}$ quantum wells with the boron $\delta$ doping in quantum wells.

\section{RESULTS AND DISCUSSION}

At $15 \mathrm{~K}$, the absorption spectra can be grouped into three regions, including a 1.8-2.37 $\mu \mathrm{m}$ peak (the inset of Fig. 2), a 3.7-6 $\mu \mathrm{m}$ peak, and a 6-16 $\mu \mathrm{m}$ peak (Fig. 2). The schematic energy levels in $\mathrm{Si} / \mathrm{SiGe} / \mathrm{Si}$ QDs are shown in Fig. 3.

The $1.8-2.37 \mu \mathrm{m}$ peak results from the intervalence band transition ( $E_{1}$ in Fig. 3) between heavy hole ground

TABLE I. Condition of three different samples.

\begin{tabular}{ccccc}
\hline \hline $\begin{array}{c}\text { Sample } \\
\text { name }\end{array}$ & $\begin{array}{c}\text { Active } \\
\text { layer }\end{array}$ & $\begin{array}{c}\delta \text {-doping } \\
\text { location }\end{array}$ & $\begin{array}{c}\text { Spacer } \\
\text { width } \\
(\mathrm{nm})\end{array}$ & $\begin{array}{c}\text { Cap } \\
\text { thickness } \\
(\mathrm{nm})\end{array}$ \\
\hline$\delta$-spacer & $\begin{array}{c}20 \text { layers } \\
\text { SiGe QD }\end{array}$ & Spacer & 80 & 130 \\
$\delta$-QD & $\begin{array}{c}20 \text { layers } \\
\text { SiGe QD } \\
5 \text {-SiGe01 }\end{array}$ & QD & 80 & 130 \\
& $\begin{array}{c}5 \text { layers } \\
\mathrm{Si}_{0.9} \mathrm{Ge} \\
\mathrm{QW}\end{array}$ & QW & 90 & 135 \\
& ${ }^{0.1}$ & & & \\
\hline \hline
\end{tabular}

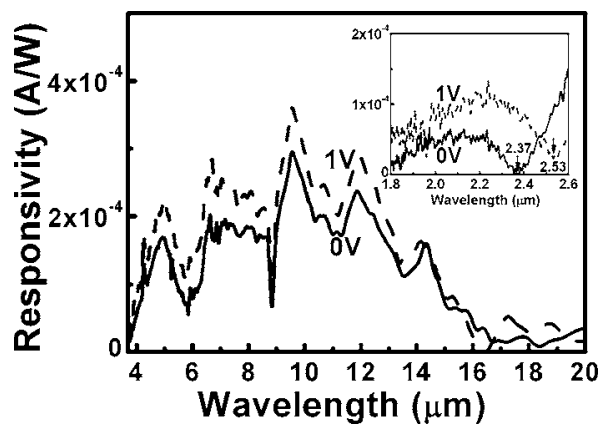

FIG. 2. Spectral response of the $\delta$-spacer sample at 0 and $1 \mathrm{~V}(15 \mathrm{~K})$. The inset shows the response at shorter wavelength, indicating cutoff wavelength at $2.37 \mu \mathrm{m}$ at $0 \mathrm{~V}$ and $2.53 \mu \mathrm{m}$ at $1 \mathrm{~V}$.

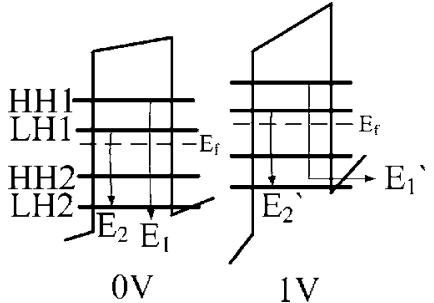

FIG. 3. The schematic energy levels in $\mathrm{Si} / \mathrm{SiGe} / \mathrm{Si}$ quantum dots under different biases. The band is not flat at $0 \mathrm{~V}$ since the flatband voltage is negative.

state (HH1) and the continuum states. When the applied voltage increases from 0 to $1 \mathrm{~V}$, the cutoff wavelength shifts from $2.37 \mu \mathrm{m}(0.52 \mathrm{eV})$ to $2.53 \mu \mathrm{m}(0.49 \mathrm{eV})$ (the inset of Fig. 2). Holes, which are excited by photons with energy less than the energy difference between the ground state and the barrier, can tunnel through the triangular barrier at $1 \mathrm{~V}$ (Fig. 3 ). The redshift is characteristic of bound-to-continuum transition. ${ }^{11,12}$

The photoluminescence (PL) spectrum at $10 \mathrm{~K}$ is shown in Fig. 4. Since the conduction band offset at the $\mathrm{Si} / \mathrm{SiGe}$ heterojunction is very small and the ground state (HH1) is very close to the valence band edge of SiGe dots, the HH1to-continuum transition energy approximately coincides with the energy gap difference between $\mathrm{Si}(1.17 \mathrm{eV})$ and $\mathrm{SiGe}$ dots $(0.7 \mathrm{eV})$.

The hole concentration of light hole ground state (LH1) is smaller than that of HH1. The LH1-to-continuum transition should be much weaker than the HH1-to-continuum transition, and no obvious absorption is observed.

The 3.7-6 $\mu \mathrm{m}$ peak results from the intersubband transition ( $E_{2}$ in Fig. 3) between the ground state of light hole (LH1) and the first excited state of light hole (LH2) in the SiGe-dot layers. The 3.7-6 $\mu \mathrm{m}$ peak does not shift as the gate voltage increases, since the LH1-to-LH2 transition (3.7-6 $\mu \mathrm{m}$ peak) occurs between two bound states, and the influence of a triangular barrier is small.

The continuous supply of holes to the $\mathrm{SiGe/Si}$ QDs is necessary when the photogenerated holes are collected by the electrode at the bottom of the substrates (Fig. 5). Two possible mechanisms could be responsible for the supply of holes. One is the electron-hole pair generation at oxide/ semiconductor interface. The electrons in the valence band jump into the conduction band via interface states, and tunnel into the gate electrode through the thin oxide $(2 \mathrm{~nm})$, and

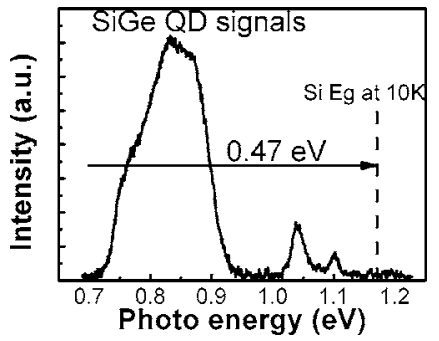

FIG. 4. The photoluminescence (PL) spectrum of the $\delta$-spacer sample at $10 \mathrm{~K}$. The band edge of the SiGe dot signal corresponding to HH1 to conduction band in $\mathrm{SiGe}$ dot is $\sim 0.7 \mathrm{eV}$. 


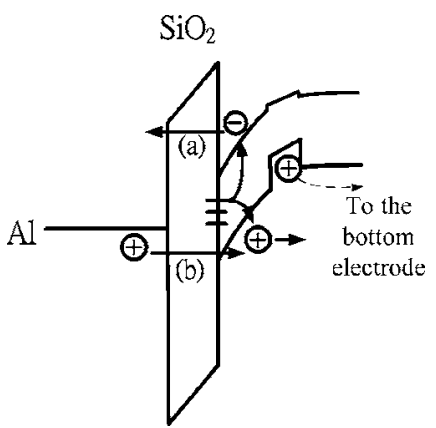

FIG. 5. Two possible mechanisms responsible for the hole supply. (a) Electrons in the valence band jump into the conduction band via interface states and tunnel into the gate electrode through the thin oxide, and the holes are left in the valence band. (b) Holes tunnel from the gate electrode to Si.

the holes are left in the valence band. The other mechanism is that holes tunnel from the gate electrode to $\mathrm{Si}$.

The $6-16 \mu \mathrm{m}$ peak mainly comes from the intraband (intersubband or intervalence band) transition in the boron $\delta$-doping wells in the Si spacers. For the shorter wavelength part in the $6-16 \mu \mathrm{m}$ peak (about $6-10 \mu \mathrm{m}$ ), there are other possible transitions in QDs such as the intersubband transition from $\mathrm{HH} 1$ to $\mathrm{HH} 2$ and the intervalence band transition from $\mathrm{HH} 1$ to LH1. However, the excited states $(\mathrm{HH} 2$ and LH1) are far from the top of the barrier, preventing photoexcited carriers to be efficiently collected. ${ }^{13}$ Therefore, the transitions (HH1 to HH2 and HH1 to LH1) may have a small contribution to the $6-10 \mu \mathrm{m}$ absorption. Moreover, oxygen with an impurity level of $160 \mathrm{meV}(7.8 \mu \mathrm{m})$ may also contribute to the $6-10 \mu \mathrm{m}$ absorption. ${ }^{14}$

For comparison, the infrared spectra of the three samples in Table I are shown in Fig. 6. The cutoff wavelengths of $\delta$-spacer and $\delta$-SiGe01 samples at the low energy end are 16 and $14 \mu \mathrm{m}$, respectively. These transitions result from the intraband transition of $\delta$-doping well (Fig. 7). The blueshift of the cutoff wavelength of the $\delta$-SiGe01 sample as compared with the $\delta$-spacer sample is probably due to the additional quantum confinement of $\mathrm{Si}_{0.9} \mathrm{Ge}_{0.1} \mathrm{QW}$ outside the $\delta$-doping well. Note that there is a valence band offset of $\sim 75 \mathrm{meV}$ between $\mathrm{Si}$ and $\mathrm{Si}_{0.9} \mathrm{Ge}_{0.1}$. The long-wavelength $(<14 \mu \mathrm{m})$ transition in the $\delta$-SiGe01 sample cannot originate from intraband transition of $\mathrm{Si}_{0.9} \mathrm{Ge}_{0.1} \mathrm{QW}$ due to the

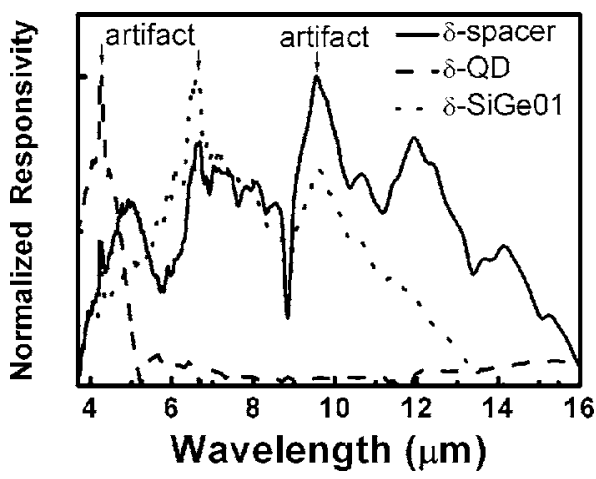

FIG. 6. Comparison of the spectral responses of the $\delta$-spacer sample, the $\delta$-QD sample, and the $\delta$-SiGe01 sample at $15 \mathrm{~K}$ (at $1 \mathrm{~V}$ bias). Both the $\delta$-spacer sample and the $\delta$-SiGe01 sample have a long-wavelength transition.

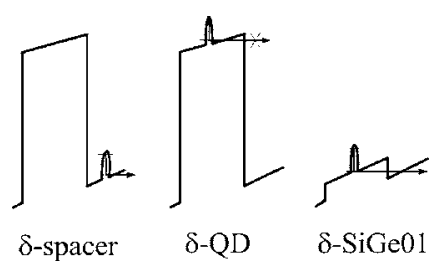

FIG. 7. The schematic detection of long-wavelength infrared inside the $\delta$-doping layer. The long-wavelength infrared photoexcited carriers can be collected in the $\delta$-spacer sample and $\delta$-SiGe01 sample. No absorption around $6-16 \mu \mathrm{m}$ is observed in the $\delta$-QD sample, since the valence band barrier of SiGe QD is too large to block the photoexcited hole from the $\delta$-doping well.

QW's shallow depth, where the cutoff wavelength should be larger than $16.5 \mu \mathrm{m}(75 \mathrm{meV})$. No absorption around $6-16 \mu \mathrm{m}$ is observed in the $\delta$-QD sample. Since the valence band barrier of SiGe QD is large enough to block the photoexcited holes from the $\delta$-doping well, the photoexcited carriers cannot be collected by the electrode (Fig. 7).

For the intersubband transition from LH1 to LH2, the cutoff wavelengths of the $\delta$-QD sample and the $\delta$-spacer sample are 5 and $6 \mu \mathrm{m}$, respectively. The smaller cutoff wavelength of the $\delta$-QD sample may be due to the many body effect, since the hole concentration in the QDs is higher. The many body effect increases the intersubband transition energy. ${ }^{15,16}$

Note that there are some artifacts, such as 2.6, 4.3, 6.6, and $9.5 \mu \mathrm{m}$ (Fig. 6), due to the absorption from the measurement environment.

The normalized detectivity $D^{*}$ is

$$
D^{*}=\frac{\sqrt{A \Delta f}}{N E P}=\frac{\sqrt{A \Delta f}}{i_{n} / R},
$$

where $A$ is the detector area $\left(3 \times 10^{-2} \mathrm{~cm}^{2}\right)$ and $\Delta f$ is the equivalent bandwidth of the electronic system. The noise equivalent power is defined as $i_{n} / R$, where $i_{n}$ is the current noise and $R$ is the responsivity. At $1 \mathrm{~V}$, the current noise is limited by the dark current and can be approximated as the shot noise $\left(2 e I_{d} \Delta f\right)^{1 / 2}$, where $I_{d}$ is the measured dark current. At $0 \mathrm{~V}$, the dark current approaches zero, and the current noise should be approximated as Johnson noise $(4 k T G \Delta f)^{1 / 2}$, where $G$ is the measured conductance.

Therefore, $D^{*}$ can be simplified as

$$
\begin{aligned}
& D^{*}=\frac{\sqrt{A} \mathrm{R}}{\sqrt{2 e I_{d}}} \text { at } 1 \mathrm{~V}, \\
& D^{*}=\frac{\sqrt{A} \mathrm{R}}{\sqrt{4 k T G}} \text { at } 0 \mathrm{~V} .
\end{aligned}
$$

Figure 8 shows the detectivity at 1 and $0 \mathrm{~V}$ bias at different temperatures. At $15 \mathrm{~K}$ and $1 \mathrm{~V}$ bias, the peak detectivity is found to be $4.3 \times 10^{8} \mathrm{~cm} \mathrm{~Hz}^{1 / 2} / \mathrm{W}$ at $9.6 \mu \mathrm{m}$ and $2.6 \times 10^{8} \mathrm{~cm} \mathrm{~Hz}^{1 / 2} / \mathrm{W}$ at $4.9 \mu \mathrm{m}$. The peak detectivity increases to $3.9 \times 10^{9} \mathrm{~cm} \mathrm{~Hz}^{1 / 2} / \mathrm{W}$ at $9.6 \mu \mathrm{m}$ and 2.3 $\times 10^{9} \mathrm{~cm} \mathrm{~Hz}^{1 / 2} / \mathrm{W}$ at $4.9 \mu \mathrm{m}$ when the bias decreases from 1 to $0 \mathrm{~V}$. Note that the conductance measured at $15 \mathrm{~K}$ is 2 $\times 10^{-7} \mathrm{~S}$. The detectivity decreases as operating temperature increases. The values of detectivity are very low in compari- 


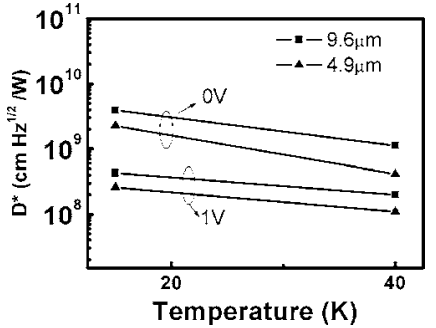

FIG. 8. The detectivity of the $\delta$-spacer sample at different temperatures. The 3.7-6 $\mu \mathrm{m}$ detection has a peak at $4.9 \mu \mathrm{m}$, and the $6-16 \mu \mathrm{m}$ detection has a peak at $9.6 \mu \mathrm{m}$.

son with that of commercialized detectors. Further optimization should be done for higher detectivity. Dark current reduction and responsivity enhancement may be the effective steps to improve the detectivity. The dark current could be reduced with a passivation technique to decrease the interface states at $\mathrm{Si} / \mathrm{LPD} \mathrm{SiO}_{2}$ interface. Antireflection coating could be used in order to increase the responsivity.

Under negative bias, the electrons in $\mathrm{Al}$ gate tunnel to the semiconductor, and this large dark current prevents the detector operation at the negative bias (Fig. 9).

\section{CONCLUSIONS}

There are three detection regions for the QDIP with $\delta$ doping in the $\mathrm{Si}$ spacer, including 1.8-2.37, 3.7-6, and 6-16 $\mu \mathrm{m}$. The $1.8-2.37 \mu \mathrm{m}$ peak results from the intervalence band transition from HH1 to the continuum states in the SiGe-dot layers. The 3.7-6 $\mu \mathrm{m}$ peak results from the inter-

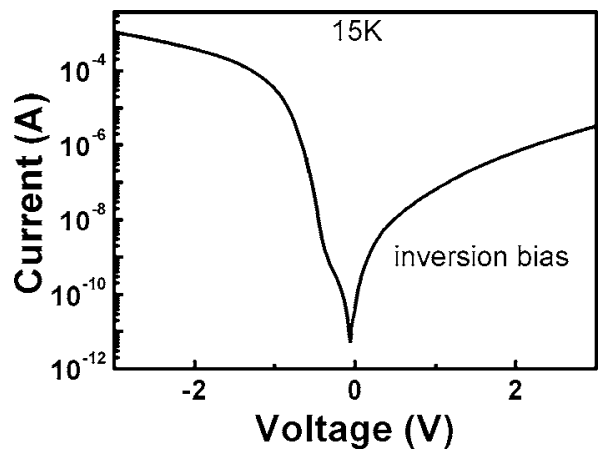

FIG. 9. Dark current of the $\delta$-spacer sample at $15 \mathrm{~K}$. The detector is operated at inversion bias. subband transition from LH1 to LH2 in the SiGe-dot layers. The cutoff wavelengths of the LH1-to-LH2 transition of the $\delta$-QD and $\delta$-spacer samples are 5 and $6 \mu \mathrm{m}$, respectively. The blueshift of the $\delta$-QD sample may be due to the many body effect. The intraband transition in $\delta$-doping quantum wells contributes to long-wavelength infrared detection $(6-16 \mu \mathrm{m})$. The cutoff wavelengths at the low energy end of $\delta$-spacer and $\delta$-SiGe01 samples are 16 and $14 \mu \mathrm{m}$, respectively. The blueshift of the cutoff wavelength of the $\delta$-SiGe01 sample is probably due to the additional quantum confinement of $\mathrm{Si}_{0.9} \mathrm{Ge}_{0.1} \mathrm{QW}$ outside the $\delta$-doping well. At $15 \mathrm{~K}$ and $0 \mathrm{~V}$, the peak detectivity is found to be 3.9 $\times 10^{9} \mathrm{~cm} \mathrm{~Hz}^{1 / 2} / \mathrm{W}$ for the $6-16 \mu \mathrm{m}$ peak and 2.3 $\times 10^{9} \mathrm{~cm} \mathrm{~Hz}^{1 / 2} / \mathrm{W}$ for the $3.7-6 \mu \mathrm{m}$ peak.

\section{ACKNOWLEDGMENTS}

This work is supported by the U.S. Air Force Office of Scientific Research/AOARD under Contract No. FA 520904P0441 and the National Science Council, Taiwan, R.O.C. under Contract nos. 95-2221-E-002-357 and 952221-E-002-370.

${ }^{1}$ P. G. Datskos, S. Rajic, L. R. Senesac, and I. Datskou, Ultramicroscopy 86, 191 (2001).

${ }^{2}$ J. Park, S.-J. Jo, S. Hong, and J.-I. Song, Solid-State Electron. 46, 651 (2002)

${ }^{3}$ G. Karunasiri, J. S. Park, J. Chen, R. Shih, J. F. Scheihing, and M. A. Dodd, Appl. Phys. Lett. 67, 2600 (1995).

${ }^{4}$ R. People, J. C. Bean, C. G. Bethea, S. K. Sputz, and L. J. Peticolas, Appl. Phys. Lett. 61, 1122 (1992).

${ }^{5}$ S. V. Bandara et al., Appl. Phys. Lett. 86, 151104 (2005).

${ }^{6}$ J.-h. Zhu, D.-w. Gong, B. Zhang, F. Lu, C. Sheng, H.-h. Sun, and X. Wang, Phys. Rev. B 54, 2662 (1996).

${ }^{7}$ M. H. Liao, C.-H. Lin, C.-H. Lee, T.-H. Cheng, T.-H. Guo, and C. W. Liu, ECS Meeting, Cancun, Mexico, No. 1278 (2006).

${ }^{8}$ O. G. Schmidt and K. Eberl, Phys. Rev. B 61, 13721 (2000).

${ }^{9}$ B.-C. Hsu, S. T. Chang, C.-R. Shie, C.-C. Lai, P. S. Chen, and C. W. Liu, Tech. Dig. - Int. Electron Devices Meet. 2002, 91.

${ }^{10}$ C. W. Liu, M. H. Lee, C. F. Lin, I. C. Lin, W. T. Liu, and H. H. Lin, Tech. Dig. - Int. Electron Devices Meet. 1999, 749.

${ }^{11}$ B. F. Levine, J. Appl. Phys. 74, R1 (1993).

${ }^{12}$ V. D. Jovanovic, P. Harrison, Z. Ikonic, and D. Indjin, J. Appl. Phys. 96, 269 (2004).

${ }^{13}$ R. P. G. Karunasiri, J. S. Park, Y. J. Mii, and K. L. Wang, Appl. Phys. Lett. 57, 2585 (1990)

${ }^{14}$ S. M. Sze, Physics of Semiconductor Devices, 2nd ed. (Wiley, New York, 1985), Part 1, p. 21.

${ }^{15}$ G. Karunasiri, Jpn. J. Appl. Phys., Part 1 33, 2401 (1994).

${ }^{16}$ Z. Chen, C. M. Hu, P. L. Liu, G. L. Shi, and S. C. Shen, J. Appl. Phys. 82, 3900 (1997). 